\title{
Trial Wavefunctions for the Goldstone Mode in $v=1 / 2+1 / 2$ Quantum Hall Bilayers
}

\author{
Gunnar Möller ${ }^{1}$ and Steven H. Simon ${ }^{2}$ \\ ${ }^{1}$ TCM Group, Cavendish Laboratory, JJ Thomson Avenue, Cambridge CB3 OHE, UK \\ ${ }^{2}$ Rudolf Peierls Centre for Theoretical Physics, Oxford University, Oxford OX1 3NP, UK \\ Correspondence should be addressed to Gunnar Möller, gm360@cam.ac.uk
}

Received 12 September 2010; Accepted 28 October 2010

Academic Editor: Yogesh Joglekar

Copyright (c) 2011 G. Möller and S. H. Simon. This is an open access article distributed under the Creative Commons Attribution License, which permits unrestricted use, distribution, and reproduction in any medium, provided the original work is properly cited.

\begin{abstract}
Based on the known physics of the excitonic superfluid or 111 state of the quantum Hall $v=1 / 2+1 / 2$ bilayer, we create a simple trial wavefunction ansatz for constructing a low-energy branch of (Goldstone) excitations by taking the overall ground state and boosting one layer with respect to the other. This ansatz works extremely well for any interlayer spacing. For small $d$, this is simply the physics of the Goldstone mode, whereas for large $d$, this is a reflection of composite fermion physics. We find hints that certain aspects of composite fermion physics persist to low $d$ whereas certain aspects of Goldstone mode physics persist to high $d$. Using these results, we show nonmonotonic behavior of the Goldstone mode velocity as a function of $d$.
\end{abstract}

The $v=1 / 2+1 / 2$ quantum Hall bilayer is a remarkably rich system $[1,2]$. At small enough spacing between the layers, $d$, the system is known to be an excitonic superfluid [3] known sometimes as the 111 phase [4]. At larger layer spacing, a phase transition or crossover is observed experimentally [511] leading to a compressible phase which is well described by two weakly coupled composite fermion Fermi liquids. The nature of this crossover, as well as whether there are intervening phases between small and large $d$, has been a matter of some debate in the community [12-20].

There are some results, however, that are extremely well established theoretically. In the limit where $d$ becomes very small, it is known that the Halperin 111 trial wavefunction becomes exact [4]. In a more BCS-like language, this wavefunction can be expressed as [3]

$$
|111\rangle=\prod_{X}\left(c_{X, \uparrow}^{\dagger}+c_{X, \downarrow}^{\dagger}\right)|0\rangle
$$

where $\uparrow$ and $\downarrow$ indicate the layer index (we assume the real spin is frozen throughout this paper) and $X$ constitutes the orbital index within the lowest Landau level (chosen to be the $x$-directed momentum in Landau gauge, e.g.). (Strictly speaking this second quantized form of the wavefunction must be projected to fixed number of particles within each layer to generate a Halperin 111 wavefunction. However, in the thermodynamic limit these two descriptions are essentially equivalent.)

The BCS-like form of (1) allows one to consider long wavelength Goldstone excitations of the form $[3,21]$

$$
\mid 111 \text { - excitation, } k\rangle=\prod_{X}\left(c_{X, \uparrow}^{\dagger}+e^{i k X} c_{X, \downarrow}^{\dagger}\right)|0\rangle .
$$

These modes are expected to form a linearly dispersing low energy branch with energy proportional to $k$ for small $k$. Physically, this Goldstone mode corresponds to superflowone layer being boosted with respect to the other. Both a linearly dispersing mode [22] and excitonic superflow $[7,9,23-25]$ were observed experimentally in this system. Some properties of the Goldstone mode were discussed in a numerical study on the torus [17].

Away from the $d \rightarrow 0$ limit, the form of the bilayer ground state is not known exactly. However, so long as we remain in the same phase of matter, there will continue to be a linearly dispersing Goldstone mode in the long wavelength limit. An approximate expression for this Goldstone mode can be obtained from the ground state wavefunction at any $d$ simply by boosting one layer with respect to the other. One purpose of the current paper is to test this technique 
of generating trial wavefunctions for the long wavelength Goldstone modes.

We note that this technique is not expected to be exact away from $d=0$, but for small $d$ is expected to be quite accurate. In a conventional picture of superfluidity, one might imagine that it would be better to find a way to boost the superfluid fraction while leaving the "normal" fraction unboosted (only at $d=0$ is the system entirely super in some sense [13]). Nonetheless, our technique appears to work quite well even away from $d=0$.

One might expect that once the system is no longer in the 111 phase of matter (roughly $d>1.5$ magnetic lengths), our technique for generating excited states would fail. However, this turns out not to be the case. First of all, at intermediate $d$ there may exist an interlayer paired state as discussed in [19]. Such a paired state would also have a Goldstone mode that could be generated from the ground state by boosting one layer in exactly the same way.

However, even at very large $d$ when either such a pairing phase is absent or pairing is extremely weak, our scheme for generating excited states still works surprisingly well. To understand why this is so, we realize that at large enough $d$ each layer is essentially independent. To the first approximation, each layer forms a composite fermion Fermi liquid, which for finite-size system has finite momentum (except when the number of electrons exactly fills a shell). The two Fermi liquids are weakly coupled and can combine their momenta to form an overall zero momentum ground state, but since the coupling between the two layers is weak, it costs very little energy to form a state of overall finite momentum instead-which can be interpreted as boosting one layer with respect to the other in comparison to the ground state.

In the absence of any superfluid order parameter (at large $d$ ), it is probably not strictly appropriate to refer to this low-energy mode as a Goldstone mode. However, since this mode may evolve continuously into the Goldstone mode at smaller $d$, we will abuse nomenclature and continue to call it a Goldstone mode (If, as conjectured in [19], the bilayer is actually paired out to large $d$, then the usage remains correct).

Throughout this paper we will work with a spherical geometry. In this case, boosting one layer with respect to the other corresponds to applying the angular momentum raising operator $L_{+}$to one layer but not the other (call this operator $L_{+, \uparrow}$ meaning that it is applied to the $\uparrow$ layer only). In the appendix we show that if we start with any $L=0$ state of the entire system, applying $\left(L_{+, t}\right)^{J}$ generates a bilayer state with overall angular momentum $L=L_{z}=J$. Our technique is then to use exact diagonalization to generate the $L=0$ ground state of the bilayer system, which is used to obtain the trial wavefunction for the excited state

$$
|\operatorname{Trial}(d): L=J\rangle=\left(L_{+, \uparrow}\right)^{J}|\operatorname{Ground} \operatorname{State}(d): L=0\rangle .
$$

Despite the apparent asymmetry between spins $\uparrow$ and $\downarrow$, the trial state $(3)$ has a distinct parity of $(-1)^{J}$ under spin reversal, as shown in the appendix. In turn, we compare this trial state to the exact excited states with angular momentum $L=J$.

Our numerical work is based on exact diagonalization of the Coulomb Hamiltonian for a bilayer system on the sphere [26]. We simplify the problem to exclude issues related to a finite tunneling amplitude between the layers, Landau level mixing, or spin (which we assume is polarized) and model each layer as an ideal 2D plane without considering its width into the third dimension. At fillings smaller than one per layer, the Hamiltonian is thus given by the projection of the Coulomb interaction into the lowest Landau level

$$
\mathcal{H}[d]=\sum_{\substack{\sigma=\uparrow, \downarrow \\ i<j}} \frac{e^{2}}{\epsilon\left|r_{\sigma, i}-r_{\sigma, j}\right|}+\sum_{i, j} \frac{e^{2}}{\epsilon \sqrt{\left|r_{\uparrow, i}-r_{\downarrow, j}\right|^{2}+d^{2}}},
$$

where sums run over all particles with the given pseudospin. The interactions are parametrized by the layer separation $d$ that is measured in units of the magnetic length $\ell_{0}=\sqrt{\hbar c / e B}$. All lengths given in this paper should be understood to be measured in units of $\ell_{0}$, where this is not explicitly indicated.

In our exact diagonalization calculations, we focus specifically on the density-balanced bilayer system with $N_{\uparrow}=N_{\downarrow}=$ $N / 2$ and devote the majority of this paper to the discussion of the state at the shift of the 111 state, namely, $N_{\phi}=$ $N-1$. We have also tested the system at the neighbouring shifts of $N_{\phi}=N$ and $N_{\phi}=N-2$ and found in these cases that the low-lying spectrum is very flat, and that the ground state may occur at finite $L$-unlike the behaviour expected from the collective modes of a condensate. We thus focus on $N_{\phi}=N-1$, but we should caution that our analysis does not exclude the possibility that for large $d$ a first-order phase transition into a state occurring at a different shift may occur. For our discussion, we obtain the two lowest-lying eigenvalues and eigenvectors in each sector of angular momentum. This is most easily achieved using a projected Lanczos algorithm [27] which uses an additional projection to the lowest energy subspace of minimal angular momentum after each multiplication with the Hamiltonian. In a given sector with fixed $L_{z}$, this procedure therefore directly yields eigenstates of $L^{2}$ with the eigenvalue $L=L_{z}$.

To assess the accuracy of the trial states (3) for the Goldstone mode, we consider their overlap with the lowest energy state in each sector of angular momentum $L^{2}$ and for layer separations $d=0 \cdots 3 \ell_{0}$, in steps of $(1 / 2) \ell_{0}$ (We will attempt to keep the convention of using the word "ground state" to indicate the absolute lowest energy state of the system, whereas the lowest energy state of an angular momentum sector will be referred to as such.) We note again that the the trial states are generated by applying the operator $\left(L_{+, \uparrow}\right)^{J}$ to the exact ground state at $L=0$. Only at $d=0$ is an exact analytical expression of the ground state known: the 111 state. At other values of $d$, the numerical ground states from exact diagonalization are used, although very accurate trial wavefunctions are also known [20]. The results are summarized in Figure 1, which also indicates overlaps with the first excited state in addition to the overlaps with the lowest energy state in each sector. At $d=0$, the ansatz 

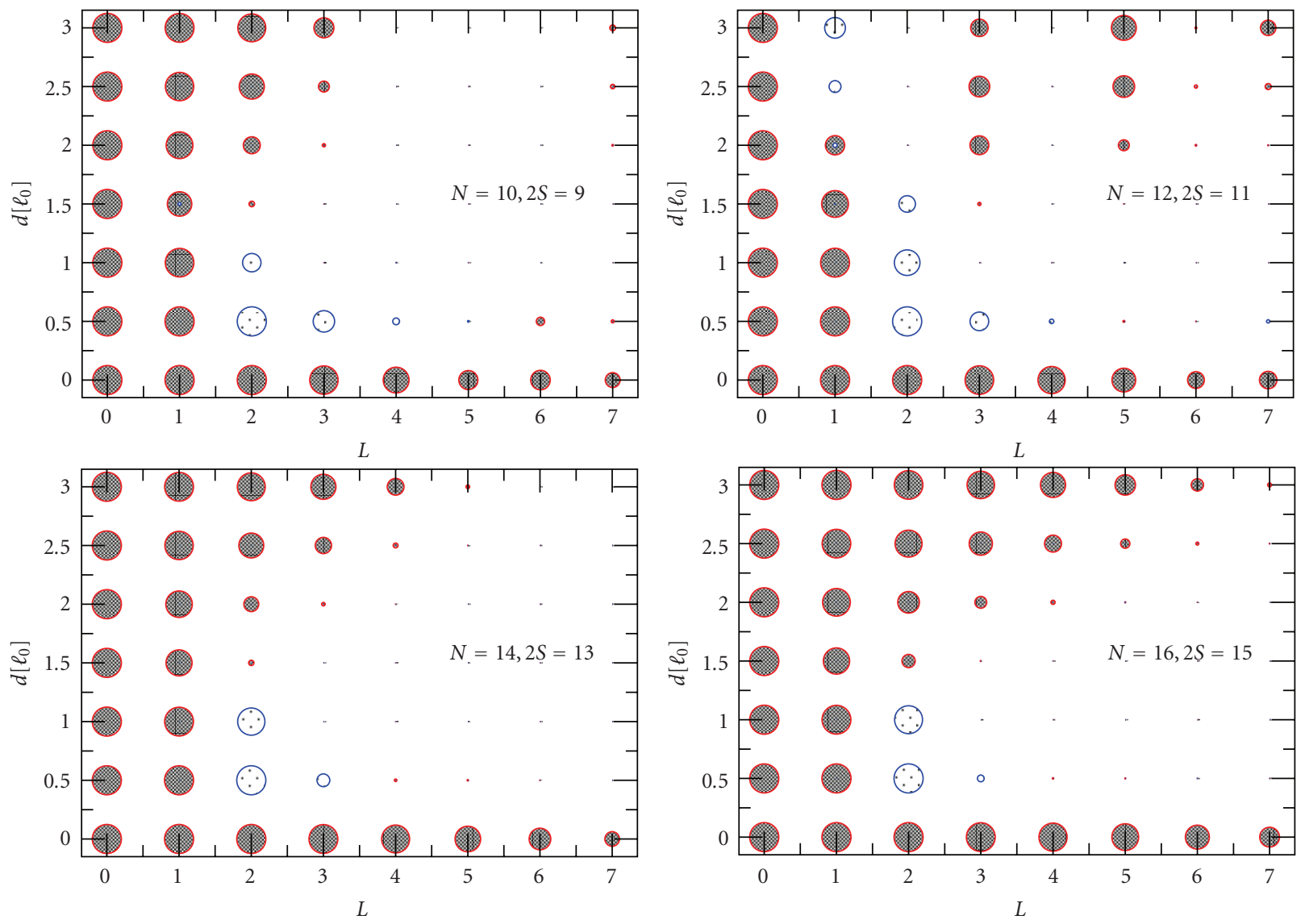

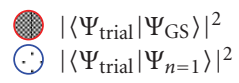$$
\bigodot \begin{array}{ll}
\odot & \left|\left\langle\Psi_{\text {trial }} \mid \Psi_{\mathrm{GS}}\right\rangle\right|^{2} \\
\hdashline & \left|\left\langle\Psi_{\text {trial }} \mid \Psi_{n=1}\right\rangle\right|^{2}
\end{array}
$$

Figure 1: Overlaps of the trial states (3) with the exact eigenstates of the Coulomb Hamiltonian for a selection of system sizes $N=10$, 12,14 , and 16 on the sphere. The magnitude of the overlap of the trial state with the exact ground state is indicated by the size of red dark shaded circles. Blue lightly shaded circles additionally indicate the overlap of the same trial states with the first excited state of the exact spectrum. Overlaps at $L=0$ are equal to one by definition and give the overall scale. The trial states are very accurate at $d=0$, giving a good description of the lowest energy mode in each sector of angular momentum (the Goldstone mode) up to large $L$. At finite layer separation $d$, the Goldstone mode is always present at small $L$ but does not reach to similarly high values of angular momentum. The description is again more successful at very large $d$.

(3) is very successful, describing excited states up to high angular momentum accurately. This is shown in more detail in Figure 2(a), which displays the magnitude of the overlap as a function of wavevector $k$. Surprisingly, for $d=0$, the overlap is very consistent with system size at given $k$, even though the Hilbert space dimension increases strongly with $N$. A very good description with overlaps above 0.8 is given up to a wavevector of $k \sim 2 \ell_{0}^{-1}$. Turning back to Figure 1 , we now focus on the overlaps at finite values of layer separation. At $d=0.5 \ell_{0}$, our trial states obtain significant overlaps only with the first excited state at $L=1$. This is not due to a disappearance of the linearly dispersing mode, however. Rather, a level crossing appears with distinct excitations occurring at energies less than those of the Goldstone mode, as can be seen from the significant overlaps with the first excited-excited state in the sector of $L=2$. At $N=12$, the crossing of the $L=2$ (3) eigenstates with the Goldstone mode occurs near $d / \ell_{0}=0.35(0.45)$. For layer separations below the first level crossing, the overlaps of the trial states with the exact eigenstates drop only slightly. Once again considering $N=12$, the overlap of the first four eigenstates remains of the order of 0.9 for $d=0.3 \ell_{0}$, and for $L=5$ it drops from 0.81 to 0.58 . At $d>1.5 \ell_{0}$ this overlap with the first excited state again disappears, signalling the presence of additional low-lying excitations of a nature different from the linearly dispersing Goldstone mode.

Finally, at the largest value of $d=3 \ell_{0}$, the ansatz for the boosted trial wavefunctions becomes more accurate than at intermediate $d$, signalling the possible emergence of a distinct mode of low-lying excitations. Based on the overlap with the trial states, we can point out that there are strong finite-size effects in the physics at large $d$. As with $d=0$, Figure 2(b) displays the numerical values of the overlaps at $d=3$. These data single out the system with $N=12$ particles as particularly poorly described by these trial states. Here, the physics at large $d$ is clearly dominated by the shell filling 


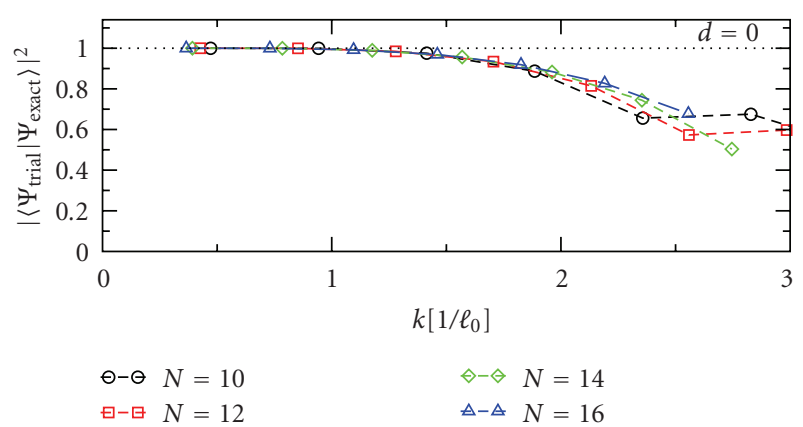

(a)

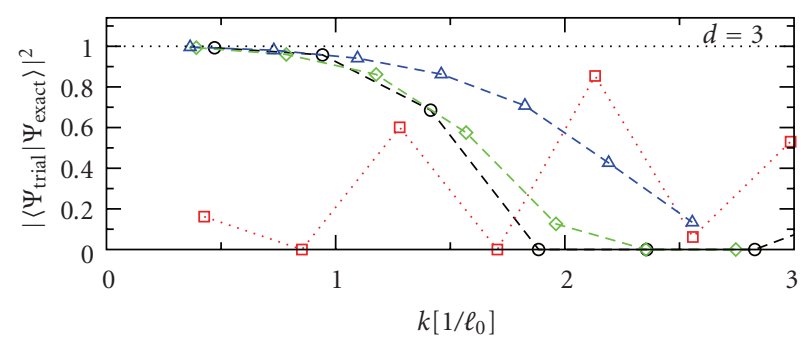

(b)

Figure 2: Overlaps of the trial states for the Goldstone mode at $d=0$ (a) and $d=3$ (b) with the exact state, as in Figure 1, plotted here as a function of wavevector $k \sim L / \sqrt{N_{\phi} / 2}$. Despite the size of the Hilbert spaces increasing significantly between the smallest and largest system shown, the overlap remains roughly constant or maybe slightly increases with $N$. The region of high overlap extends roughly up to $k \approx 2 \ell_{0}^{-1}$. The failure of this approach for $d=3$ and $N=12$ is discussed in detail in the text.

effects of composite fermions. As $N_{\uparrow}=N_{\downarrow}=6$ electrons per layer precisely fill the lowest two shells of composite fermion orbitals in one quantum of effective flux, this system size is aliased with the situation where each layer forms its own incompressible $v=2 / 5$ state with angular momentum zero in each layer-making it impossible to form higher angular momenta states by boosting one layer with respect to the other.

Before proceeding further, note the rather unusual feature that, excepting $N=12$, the trial states give higher overlaps for larger systems at $d=3 \ell_{0}$. This unusual behaviour is related to a different manifestation of the composite fermion shell filling effect. As we have shown in [19], the ground state at large layer separation is a state in which each layer individually obeys Hund's rule and maximizes the angular momentum per layer $L_{\sigma}=L_{\uparrow}=L_{\downarrow}$, while both layers are combined into a total $L=0$ state. Without modifying the correlations inside each layer, the same states with $L_{\sigma}$ per layer can be paired into excited states with subsequently larger angular momenta, up to a maximum $L_{\max }=2 L_{\sigma}$. For the system sizes with partially filled composite fermion shells in each layer, one obtains the values of $L_{\sigma}=3 / 2$ for $N=10, L_{\sigma}=5 / 2$ for $N=14$, and $L_{\sigma}=3 / 2+5 / 2=4$ for $N=16$ particles. We therefore expect a low-lying mode of excitations with angular momenta up to
$L_{\max }=3, L_{\max }=5$, and $L_{\max }=8$, respectively. Indeed, upon inspection of the spectra, such a mode can be identified.

As an example, Figure 3 displays the spectra for the system with $N=16$ particles, including different values of the layer separation. Indeed for $d=3 \ell_{0}$, shown in the bottom right panel, there is clear evidence of a mode of excitations terminating at $L=8$. Its dispersion is approximately linear at small $L$; however, it has a quadratic component as well. This should be compared to the Goldstone mode at $d=0$ (top left panel), for which linear dispersion is clearly realized up to high values of angular momentum.

Once the termination of the low-energy branch at $L_{\max }=$ 8 is identified at large $d$, it becomes apparent that this feature of a jump in the spectrum at $L_{\max }$ exists at all values of layer separation shown, with the exception of the $S U(2)$ invariant case of vanishing $d$. Note that this termination is a feature of composite fermion physics, explained by successively filling the lowest shells of these composite particles, while obeying Hund's rule. The observation that composite fermion physics intervenes at very small layer separation had been made previously by the current authors. While the 111 state can be regarded as a condensate of composite bosons, it was shown that an accurate description of the ground state requires a mixed-fluid description of both composite bosons and composite fermions at any finite layer separation $[16,20]$. The identified jump may constitute evidence for the mixedfluid picture in the excitation spectrum, but more study of these excitation spectra will certainly be required.

A linearly dispersing mode at small $k$ exists at all values of the layer separation. The states which were shown in Figure 1 to have large overlap with the trial states (3) are highlighted by blue circles in the spectra shown in Figure 3. These states very accurately come to lie on a single line, which is true especially for the 111 state at $d=0$, but also for the intermediate layer separations such as $d=1 \ell_{0}$, where the first excited state at $L=2$ lies in the continuation of the line through the points at $L=0$ and $L=1$ and is shown to be associated to the Goldstone mode by its overlap. Judging by the spectra, it is also very suggestive that multiple level crossings occur at larger values of $L$, for example, at $L=2$ for $d=1.5 \ell_{0}$. Finally, between $d=2 \ell_{0}$ and $d=3 \ell_{0}$, a change occurs in the association of the low-lying mode with the Goldstone mode trial states. For $d=2 \ell_{0}$, only the states up to $L=2$ have a good overlap, and the remaining states of the already well-formed band of low-lying states in the exact spectrum are of a different nature. The transition to low overlaps occurs at a point where this band has a visible kink and flattens out. Finally at $d=3 \ell_{0}$, this low-lying band has good overlaps up to much higher momenta, which, as we have discussed above, is a reflection of two approximately uncoupled composite fermion Fermi seas which each maximizes its own angular momentum according to Hund's rule.

Given the existence of a linearly dispersing mode over the whole range of layer separations, we now consider how its velocity changes with $d$. Comparing the results obtained for different system sizes, a relatively strong dependence of the velocity $v=\partial E / \partial k$ is evident. Applying charging corrections to take account of the shift in the charge density of the 

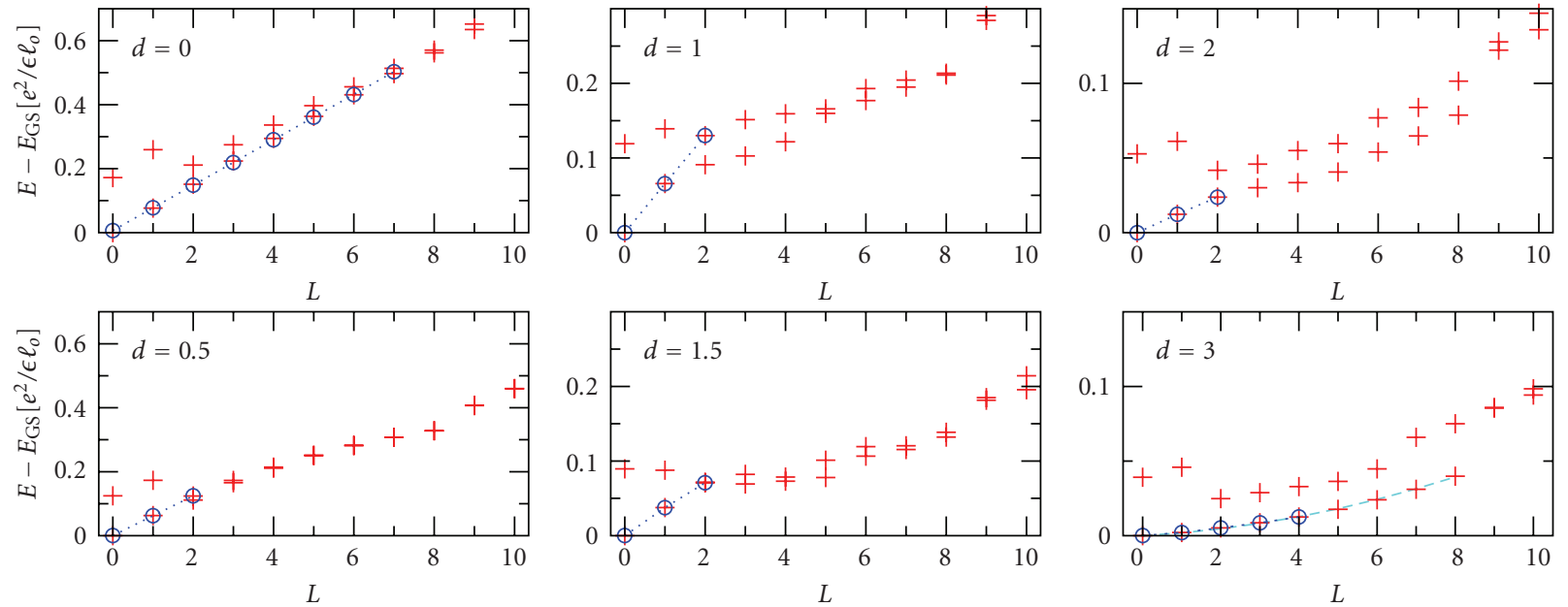

Figure 3: Spectra of the Coulomb Hamiltonian in the bilayer system on the sphere for $N=16$ particles with $N_{\phi}=15$ flux, for layer separations $d$ ranging from $d=0$ (top left) to $d=3 \ell_{0}$ (bottom right). Energies are indicated in units of $e^{2} / \epsilon \ell_{0}$ relative to the ground state energy $E_{\mathrm{GS}}$ at $L=0$. Red crosses mark the lowest two eigenstates in each sector of given $L$. Blue circles indicate those states which were identified as part of the Goldstone branch by high overlaps (see Figure 1). At $d=0$, the Goldstone mode is very well formed, while it is clearly visible at small and intermediate $d$ how there are level crossings with additional low lying excitations. At $d=3$, a mode of low-lying excitations is once again clearly separated from the rest of the spectrum, which terminates sharply at $L=8$. Its dispersion is linear at small $L$, but it has a quadratic component responsible of the upturn at larger $L$. Note the change in the scale of the $y$-axis for the three columns of panels.

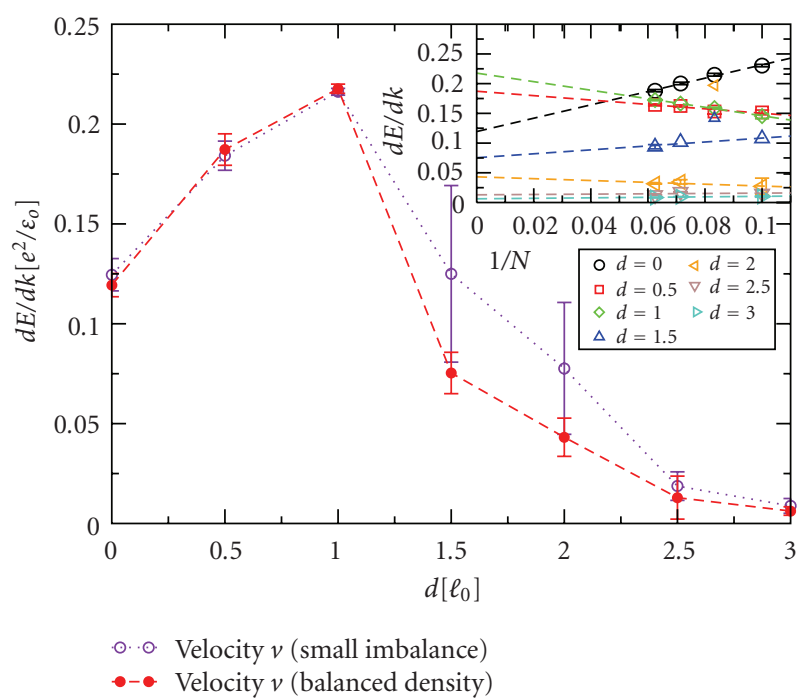

Figure 4: The velocity of the Goldstone mode depends strongly on the layer separation and peaks near $d \sim \ell_{0}$. The velocity has significant finite-size effects on the sphere, and therefore we extract an estimate of its value in the thermodynamic limit by extrapolating the finite-size values over the inverse system size (see inset). The main figure shows the resulting velocities for a density balanced bilayer, as well as for a slightly imbalanced system, as discussed in the main text.

system [28] does not suffice to absorb these effects. Thus, in addition to measuring energies in units of the rescaled magnetic length $\ell_{0}^{\prime}=\sqrt{v N_{\phi} / N} \ell_{0}$, we analyze the scaling of the mode velocity as a function of the inverse system size $N^{-1}$. These scalings are fitted well by linear extrapolation to the thermodynamic limit, as shown in the inset of Figure 4. The data for $N=12$ and large layer separation are easily identified as outliers, due to the shell filling effects discussed above. Generally, the slope is decreasing with system size. However, at small finite layer separations the opposite scaling takes effect. The resulting estimates for the mode velocity in the thermodynamic limit shown in the main graph of Figure 4 therefore show a pronounced maximum near $d=$ $\ell_{0}$, which has about twice the magnitude as the value found at $d=0$. Beyond this point, the mode velocity decreases monotonically and drops to about $1 / 10$ th the value of the 111 state at layer separation $d=3 \ell_{0}$. In Figure 4 , we have also explored how the velocity is affected when we depart from the case of completely balanced densities in both layers. When an additional particle is added to one of the layers, we continue to find a well-defined Goldstone mode across the full range of layer separations, and the velocity extrapolates to the same values for small $d$ and in the limit of large $d$. However, in the region around $d \sim 1.5 \ell_{0}$ where the vanishing of the condensate fraction indicates a phase transition, the velocity extrapolates to slightly higher values for the imbalanced case, that is, the lowest-lying excitations occur at higher energy. This may be indicative of the presence of pairing in this range of layer separations [19]. On the torus, a low-lying state has been found, which was interpreted as a pseudospin spiral state [17]. However, this mode does not occur in the spherical geometry. Further investigation will be needed to understand this difference.

A previous experiment [22] has probed the velocity of the neutral mode at layer separations near the transition into the incompressible phase at large $d$, obtaining a combined best fit of $v=1.4 \times 10^{4} \mathrm{~m} / \mathrm{s}$ for data at the three layer separations $d_{1}=1.61 \ell_{0}, d_{2}=1.71 \ell_{0}$, and $d_{3}=1.76 \ell_{0}$. 
Based on linear extrapolation between our numerical data at $d=1.5 \ell_{0}$ and $d=2 \ell_{0}$, the corresponding estimates are $v\left(d_{1}\right)=1.14 \times 10^{4} \mathrm{~m} / \mathrm{s}, v\left(d_{2}\right)=1.21 \times 10^{4} \mathrm{~m} / \mathrm{s}$, and $v\left(d_{3}\right)=$ $1.33 \times 10^{4} \mathrm{~m} / \mathrm{s}$, all slightly smaller but within about $20 \%$ of the proposed fit to the experimentally obtained values. Had the data in [22] been fitted separately at each layer separation, the velocity at $d=1.71 \ell_{0}$ would have been estimated to be about $10 \%$ smaller than that at $d=1.61 \ell_{0}$, roughly reflecting the ratio of our predicted values. The data at $d=1.76 \ell_{0}$ appears to be rather noisier, probably due to the vicinity to the phase transition, and would be difficult to fit on its own. We suggest that a significant enhancement of the linear mode velocity should be seen deeper inside the interlayer coherent phase at smaller layer separation.

To summarize our results, we use the ansatz (3) to construct trial wavefunctions for a low-energy branch of excitations based on the exact ground state wavefunction. This ansatz is accurate at all interlayer spacings $d$ when $k$ is small, and it is accurate at all $k$ when either $d$ is small or $d$ is large (so long as we do not have a filled shell configuration, whereupon only $k=0$ is in this low energy branch). We find hints that certain aspects of the composite fermion physics persist to low $d$ whereas certain aspects of the Goldstone mode physics persist to high $d$. Applying these results to the analysis of our numerical data, we show nonmonotonic behavior of the Goldstone mode velocity as a function of the layer separation $d$. It would be interesting to look for this nonmonotonicity of the Goldstone mode velocity experimentally.

\section{Appendix}

\section{Angular Momentum of Two Coupled Subsystems}

In this appendix, we will use the standard angular momentum notation $|l, m\rangle$ to indicate eigenstates of the $L^{2}$ and $L_{z}$ operators.

We consider two subsystems $\uparrow$ and $\downarrow$ with corresponding angular momentum operators $\mathbf{L}_{\uparrow}$ and $\mathbf{L}_{\downarrow}$. These two subsystems combine to form the total system with angular momentum operator

$$
\mathbf{L}=\mathbf{L}_{\uparrow}+\mathbf{L}_{\downarrow}
$$

Our objective is to show that given an eigenstate of the total system with $|l=0, m=0\rangle$ application of $\left(L_{+1}\right)^{J}$ to this system will produce an eigenstate of the total system with $|l=J, m=J\rangle$. To achieve this, it is sufficient to show that

$$
L_{+}|J, J\rangle \sim|J+1, J+1\rangle
$$

Obviously, applying $L_{++}$to any state increments its overall $L_{z}$ eigenvalue by one (or kills the state), so all that remains is to show that applying $L_{++}$to $|J, J\rangle$ results in an eigenstate $L=J+1$ of $L^{2}$, that is, results in an eigenvalue of $L^{2}$ being given by $(J+1)(J+2)$. that

Using (A.1), it is just a matter of some algebra to show

$$
\begin{aligned}
{\left[L_{+\uparrow}, L^{2}\right] } & =2 L_{z \uparrow} L_{+\downarrow}-2 L_{+\uparrow} L_{z \downarrow} \\
& =2\left(L_{+}-L_{+\uparrow}\right)\left(L_{z}+1\right)-2 L_{z \downarrow} L_{+} .
\end{aligned}
$$

We then apply both sides of this equation to the state $|J, J\rangle$. Noting that $L_{+}$kills $|J, J\rangle$, we obtain

$$
\begin{aligned}
L^{2}\left[L_{+\uparrow}|J, J\rangle\right] & =\left[L_{+\uparrow}(J(J+1))+2 L_{+\uparrow}(J+1)\right]|J, J\rangle \\
& =(J+1)(J+2)\left[L_{+\uparrow}|J, J\rangle\right],
\end{aligned}
$$

which completes the proof.

From the particular form of the eigenstate, it further follows that it has a parity of $(-1)^{J}$ under reversal of the $z$ component of (pseudo-)spin. Noting that

$$
L_{+, \text {total }}\left|L_{\text {total }}=L_{z \text {,total }}=J\right\rangle=0,
$$

and since $L_{\text {total }}=L_{+, 1}+L_{+, \downarrow}$, we have $L_{+, \uparrow}=-L_{+, \downarrow}$ when applied to any state of the form $\left|L_{\text {total }}=L_{z \text {,total }}=J\right\rangle$ for any $J$. The trial state (3) can thus equivalently be obtained by applying $L_{+, \downarrow}^{J}$, since the state $|J, J\rangle$ is unique.

\section{Acknowledgment}

G. Möller acknowledges support from Trinity Hall Cambridge, as well as from the Institute of Complex Adaptive Matter (ICAM). SHS thanks the Aspen Center for Physics for its hospitality.

\section{References}

[1] S. Das Sarma and A. Pinczuk, Eds., Perspectives in Quantum Hall effects, Wiley, New York, NY, USA, 1997, see chapter 2 by J. Eisenstein and chapter 5 by S. M. Girvin and A. H. MacDonald.

[2] J. P. Eisenstein and A. H. MacDonald, "Bose-Einstein condensation of excitons in bilayer electron systems," Nature, vol. 432, no. 7018, pp. 691-694, 2004.

[3] K. Moon, H. Mori, K. Yang et al., "Spontaneous interlayer coherence in double-layer quantum Hall systems: charged vortices and Kosterlitz-Thouless phase transitions," Physical Review B, vol. 51, no. 8, pp. 5138-5170, 1995.

[4] B. I. Halperin, "Theory of the quantized Hall conductance," Helvetica Physica Acta, vol. 56, p. 75, 1983.

[5] S. Q. Murphy, J. P. Eisenstein, G. S. Boebinger, L. N. Pfeiffer, and K. W. West, "Many-body integer quantum Hall effect: evidence for new phase transitions," Physical Review Letters, vol. 72, no. 5, pp. 728-731, 1994.

[6] I. B. Spielman, J. P. Eisenstein, L. N. Pfeiffer, and K. W. West, "Resonantly enhanced tunneling in a double layer quantum hall ferromagnet," Physical Review Letters, vol. 84, no. 25, pp. 5808-5811, 2000.

[7] E. Tutuc, M. Shayegan, and D. A. Huse, "Counterflow measurements in strongly correlated GaAs hole bilayers: evidence for electron-hole pairing," Physical Review Letters, vol. 93, no. 3, Article ID 036802, 2004.

[8] R. D. Wiersma, J. G. S. Lok, S. Kraus et al., "Activated transport in the separate layers that form the $\nu_{T}=1$ Exciton condensate," Physical Review Letters, vol. 93, no. 26, Article ID 266805, 2004. 
[9] M. Kellogg, J. P. Eisenstein, L. N. Pfeiffer, and K. W. West, "Vanishing hall resistance at high magnetic field in a doublelayer two-dimensional electron system," Physical Review Letters, vol. 93, no. 3, Article ID 036801, 2004.

[10] N. Kumada, K. Muraki, K. Hashimoto, and Y. Hirayama, "Spin degree of freedom in the $v=1$ bilayer electron system investigated by nuclear spin relaxation," Physical Review Letters, vol. 94, no. 9, Article ID 096802, 2005.

[11] A. R. Champagne, J. P. Eisenstein, L. N. Pfeiffer, and K. W. West, "Evidence for a finite-temperature phase transition in a bilayer quantum hall system," Physical Review Letters, vol. 100, no. 9, Article ID 096801, 2008.

[12] K. Yang, K. Moon, L. Belkhir et al., "Spontaneous interlayer coherence in double-layer quantum Hall systems: symmetrybreaking interactions, in-plane fields, and phase solitons," Physical Review B, vol. 54, no. 16, pp. 11644-11658, 1996.

[13] Y. N. Joglekar and A. H. MacDonald, "Microscopic functional integral theory of quantum fluctuations in double-layer quantum Hall ferromagnets," Physical Review B, vol. 64, no. 15, Article ID 155315, 10 pages, 2001.

[14] Y. B. Kim, C. Nayak, E. Demler, N. Read, and S. Das Sarma, "Bilayer paired quantum Hall states and Coulomb drag," Physical Review B, vol. 63, no. 20, Article ID 205315, 9 pages, 2001.

[15] J. Schliemann, S. M. Girvin, and A. H. MacDonald, "Strong correlation to weak correlation phase transition in bilayer quantum Hall systems," Physical Review Letters, vol. 86, no. 9, pp. 1849-1852, 2001.

[16] S. H. Simon, E. H. Rezayi, and M. V. Milovanovic, "Coexistence of composite bosons and composite fermions in $v=$ $1 / 2+1 / 2$ quantum hall bilayers," Physical Review Letters, vol. 91, no. 4, Article ID 046803, 4 pages, 2003.

[17] K. Park, "Spontaneous pseudospin spiral order in bilayer quantum Hall systems," Physical Review B, vol. 69, no. 4, Article ID 045319, 6 pages, 2004.

[18] N. Shibata and D. Yoshioka, "Ground state of $v=1$ bilayer quantum hall systems," Journal of the Physical Society of Japan, vol. 75, no. 4, Article ID 043712, 4 pages, 2006.

[19] G. Möller, S. H. Simon, and E. H. Rezayi, "Paired composite fermion phase of quantum hall bilayers at $\nu=1 / 2+1 / 2$," Physical Review Letters, vol. 101, no. 17, Article ID 176803, 2008.

[20] G. Möller, S. H. Simon, and E. H. Rezayi, "Trial wave functions for $v=1 / 2+1 / 2$ quantum Hall bilayers," Physical Review B, vol. 79, no. 12, Article ID 125106, 2009.

[21] M. Rasolt, F. Perrot, and A. H. MacDonald, "New gapless modes in the fractional quantum Hall effect of multicomponent fermions," Physical Review Letters, vol. 55, no. 4, pp. 433436, 1985.

[22] I. B. Spielman, J. P. Eisenstein, L. N. Pfeiffer, and K. W. West, "Observation of a linearly dispersing collective mode in a quantum Hall ferromagnet," Physical Review Letters, vol. 87, no. 3, Article ID 036803, 4 pages, 2001.

[23] M. Kellogg, I. B. Spielman, J. P. Eisenstein, L. N. Pfeiffer, and K. W. West, "Observation of quantized Hall drag in a strongly correlated bilayer electron system," Physical Review Letters, vol. 88, no. 12, Article ID 126804, 4 pages, 2002.

[24] M. Kellogg, J. P. Eisenstein, L. N. Pfeiffer, and K. W. West, "Bilayer quantum Hall systems at $\nu_{T}=1$ : coulomb drag and the transition from weak to strong interlayer coupling," Physical Review Letters, vol. 90, no. 24, Article ID 246801, 4 pages, 2003.

[25] E. Tutuc and M. Shayegan, "Interaction and disorder in bilayer counterflow transport at filling-factor one," Physical Review B, vol. 72, no. 8, Article ID 081307, 4 pages, 2005.

[26] F. D. M. Haldane, "Fractional quantization of the hall effect: a hierarchy of incompressible quantum fluid states," Physical Review Letters, vol. 51, no. 7, pp. 605-608, 1983.

[27] A. Wójs, G. Möller, S. H. Simon, and N. R. Cooper, "Skyrmions in the moore-read state at $v=5 / 2$," Physical Review Letters, vol. 104, no. 8, Article ID 086801, 4 pages, 2010.

[28] N. D’Ambrumenil and R. Morf, "Hierarchical classification of fractional quantum Hall states," Physical Review B, vol. 40, no. 9, pp. 6108-6119, 1989. 

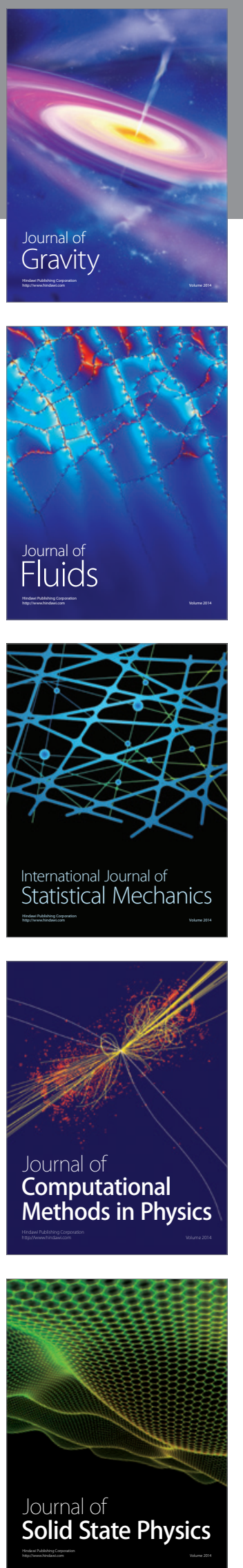

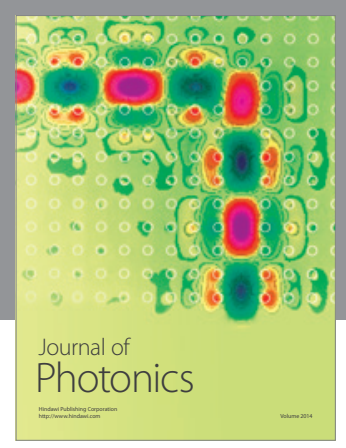

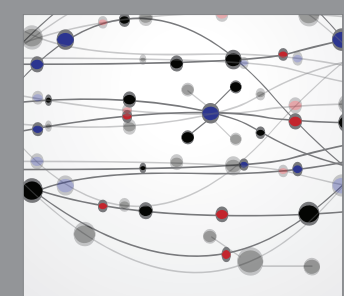

The Scientific World Journal
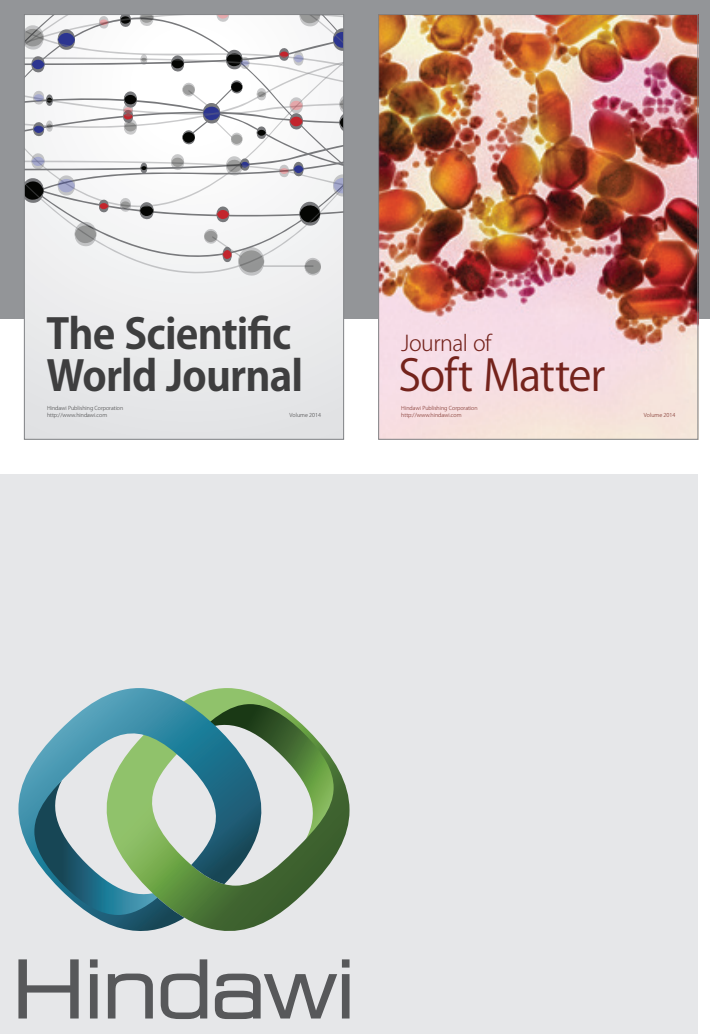

Submit your manuscripts at

http://www.hindawi.com
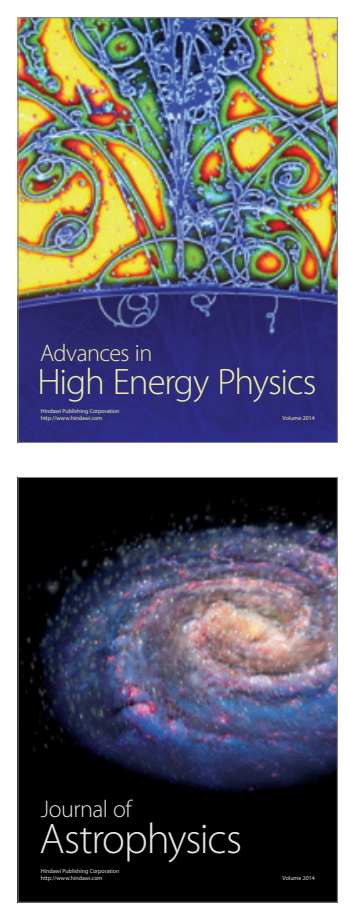
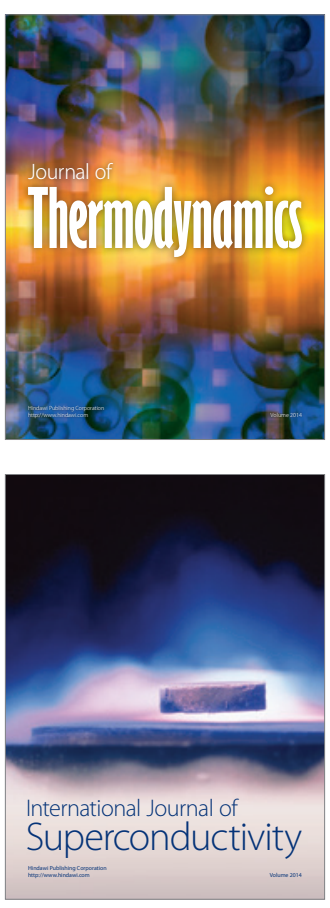
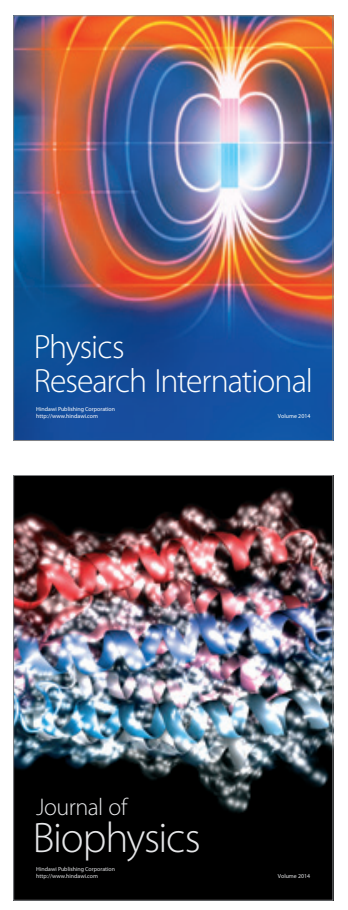
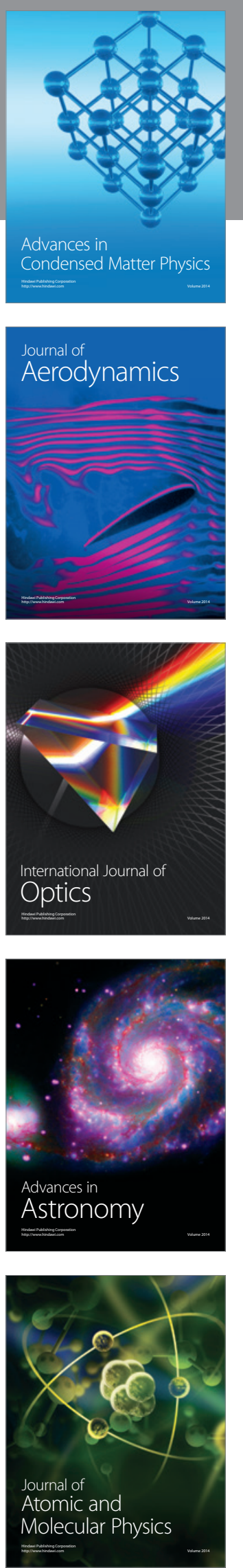